\title{
THE STRESS ON THE BOUNDARY OF AN ELASTIC HALF-PLANE IN WHICH BODY FORCES ARE ACTING
}

\author{
by IAN N. SNEDDON $\dagger$ \\ (Received 9 September, 1964)
}

1. Intrdouction. In this note we consider the problem of determining the stress on the boundary $y=0$ of the elastic half-plane $y \geqq 0$ when there are prescribed body forces acting in the interior and the boundary is free from applied stress. Expressions for the components of stress at a general point of the half-plane when the imposed body force is concentrated at a single point have been derived by Melan [1], Sneddon [2] and Green [3], each author making use of a different method.

The method employed in [2] - see also pp. 415-422 of [4]-consists in superposing on the known solution of the stress field due to a point force acting at an interior point of the whole plane, a solution of the second basic problem for a half-plane which leads to the condition of zero applied stress on the boundary. This, like the other methods cited, leads to quite a lengthy calculation to determine the distribution of stress in the interior of the halfplane, but, in the analysis of practical problems in which this solution is used, most interest is attached to calculating the distribution of stress and the form of the displacement vector on the boundary. Here we make use of an elementary property of the Laplace transform to calculate these surface values.

In $\$ 2$ the basic formulae corresponding to an arbitrary distribution of body forces are derived. In the next three sections the forms of surface stress corresponding to three special cases are considered-body forces acting in a direction normal to the boundary ( $\$ 3)$, point force $(\$ 4)$ and body forces derivable from a potential function $(\$ 5)$. Finally, in $\$ 6$, expressions are derived for the components of the surface displacement.

The equations of plane strain (in the classical theory of elasticity) are assumed throughout; the solutions corresponding to a state of plane stress can easily be deduced by means of the usual trivial changes in the values of the elastic constants.

2. The basic formulae. We consider the distribution of stress in the half-plane $y \geqq 0$ when the boundary is free from applied stress and there are body forces $[X(x, y), Y(x, y)]$ acting on the body whose density is taken to be $\rho$. We assume that the components of the stress tensor all tend to zero as $r \rightarrow \infty$, where $r=\sqrt{ }\left(x^{2}+y^{2}\right), y \geqq 0$.

If we take the Fourier transforms of the equations of equilibrium

$$
\frac{\partial \sigma_{x x}}{\partial x}+\frac{\partial \sigma_{x y}}{\partial y}+\rho X=0, \quad \frac{\partial \sigma_{x y}}{\partial x}+\frac{\partial \sigma_{y y}}{\partial y}+\rho Y=0
$$

we find that

$$
\begin{aligned}
& -i \xi \Sigma_{x x}+D \Sigma_{x y}+P=0, \\
& -i \xi \Sigma_{x y}+D \Sigma_{y y}+Q=0,
\end{aligned}
$$

† Sponsored by the U.S. Joint Services Advisory Group under Contract No. AF 49(638)-1159 with the Applied Mathematics Research Group, North Carolina State, Raleigh. 
where we have written

$$
\Sigma_{x x}(\xi, y)=\mathscr{F}\left[\sigma_{x x}(x, y) ; x \rightarrow \xi\right]=\frac{1}{\sqrt{(2 \pi)}} \int_{-\infty}^{\infty} \sigma_{x x}(x, y) e^{i \xi x} d x,
$$

etc., $D$ denotes the operator $d / d y$ and we have taken

$$
\mathscr{F}[\rho X(x, y) ; x \rightarrow \xi]=P(\xi, y), \quad \mathscr{F}[\rho Y(x, y) ; x \rightarrow \xi]=Q(\xi, y) .
$$

Similarly we find that the stress-strain relations

$$
\begin{aligned}
2 \mu \frac{\partial u_{x}}{\partial x} & =(1-\eta) \sigma_{x x}-\eta \sigma_{y y}, \\
2 \mu \frac{\partial u_{y}}{\partial y} & =(1-\eta) \sigma_{y y}-\eta \sigma_{x x}, \\
\mu\left(\frac{\partial u_{y}}{\partial x}+\frac{\partial u_{x}}{\partial y}\right) & =\sigma_{x y},
\end{aligned}
$$

in which $\mu$ denotes the rigidity modulus and $\eta$ the Poisson ratio of the half-plane, are equivalent to the equations

where

$$
\begin{aligned}
-2 \mu i \xi U_{x} & =(1-\eta) \Sigma_{x x}-\eta \Sigma_{y y}, \\
2 \mu D U_{y} & =(1-\eta) \Sigma_{y y}-\eta \Sigma_{x x}, \\
\mu\left(D U_{x}-i \xi U_{y}\right) & =\Sigma_{x y},
\end{aligned}
$$

$$
U_{x}(\xi, y)=\mathscr{F}\left[u_{x}(x, y) ; x \rightarrow \xi\right], \quad U_{y}(\xi, y)=\mathscr{F}\left[u_{y}(x, y) ; x \rightarrow \xi\right] .
$$

If we make the representation

with

$$
P(\xi, y)=2 \mu i \xi \Phi(\xi, y), \quad Q(\xi, y)=2 \mu D \Psi(\xi, y),
$$

$$
\Phi(\xi, 0)=\Psi(\xi, 0)=0,
$$

we find that the equations $(2.1),(2.2),(2.4),(2.5),(2.6)$ have solution

$$
\Sigma_{x x}=2 \mu\left(D^{2} G+\Phi\right), \quad \Sigma_{x y}=2 \mu i \xi D G, \quad \Sigma_{y y}=-2 \mu\left(\Psi+\xi^{2} G\right),
$$

where the function $G(\xi, y)$ satisfies the fourth order ordinary differential equation

$$
(1-\eta)\left(D^{2}-\xi^{2}\right)^{2} G=-\left[\eta D^{2}+(1-\eta) \xi^{2}\right] \Psi(\xi, y)-\left[(1-\eta) D^{2}+\eta \xi^{2}\right] \Phi(\xi, y) .
$$

If the boundary is free from applied stress, we have the conditions

$$
\sigma_{x y}(x, 0)=0, \quad \sigma_{y y}(x, 0)=0,
$$

and it follows from equations (2.9), (2.8) that these are equivalent to the relations

$$
D G(\xi, 0)=0, \quad G(\xi, 0)=0 .
$$

D 
The values of $D^{2} G(\xi, 0) \equiv G_{2}(\xi), D^{3} G(\xi, 0) \equiv G_{3}(\xi)$ are not known but we note from the first of the equations (2.9) that

$$
\Sigma_{x x}(\xi, 0)=2 \mu G_{2}(\xi) .
$$

We solve the equation (2.10) by taking the Laplace transform of both sides and expressing the result in terms of

$$
\bar{G}(\xi, p)=\mathscr{L}[G(\xi, y) ; y \rightarrow p]=\int_{0}^{\infty} G(\xi, y) e^{-p y} d y .
$$

Because of equations (2.11) we find that

$$
\mathscr{L}\left[D^{2} G ; p\right]=p^{2} \bar{G}, \quad \mathscr{L}\left[D^{4} G ; p\right]=p^{4} \bar{G}-p G_{2}-G_{3},
$$

so that equation $(2.10)$ is equivalent to the relation

where

$$
(1-\eta)\left(p^{2}-\xi^{2}\right)^{2} \bar{G}(\xi, p)=\Lambda(\xi, p)
$$

$$
\begin{array}{r}
\Lambda(\xi, p)=(1-\eta)\left(p G_{2}+G_{3}\right)-\left[\eta p^{2}+(1-\eta) \xi^{2}\right] \bar{\Psi}(\xi, p)+\eta \Psi_{y}(\xi, 0) \\
-\left[(1-\eta) p^{2}+\eta \xi^{2}\right] \bar{\Phi}(\xi, p)+(1-\eta) \bar{\Phi}_{y}(\xi, 0) .
\end{array}
$$

Now if the components of stress are to vanish as $y \rightarrow \infty, \bar{G}(\xi, p)$ cannot have terms such as

$$
\frac{A(\xi)}{p-|\xi|}, \quad \frac{B(\xi)}{(p-|\xi|)^{2}} \text {. }
$$

In other words $p=|\xi|$ must be a double zero of $\Lambda(\xi, p)$ regarded as a function of $p$. Hence we have the conditions

$$
(1-\eta)\left(|\xi| G_{2}+G_{3}\right)=\xi^{2} \bar{\Psi}(\xi,|\xi|)-\eta \bar{\Psi}_{y}(\xi, 0)+\xi^{2} \bar{\Phi}(\xi,|\xi|)-(1-\eta) \bar{\Phi}_{y}(\xi, 0),
$$

and

$$
(1-\eta) G_{2}=2 \eta|\xi| \bar{\Psi}(\xi,|\xi|)+\xi^{2} \bar{\Psi}_{p}(\xi,|\xi|)+2(1-\eta)|\xi| \bar{\Phi}(\xi,|\xi|)+\xi^{2} \bar{\Phi}_{p}(\xi,|\xi|)
$$

If we substitute the expression for $G_{2}(\xi)$ given by this last equation into equation (2.12), we obtain the required expression for $\Sigma_{x x}(\xi, 0)$ and hence the formula

$$
\sigma_{x x}(x, 0)=2 \mu \mathscr{F}^{-1}\left[G_{2}(\xi) ; \xi \rightarrow x\right]
$$

for the determination of the surface stress.

3. Body forces normal to the boundary. An important special case arises when the body force is always normal to the boundary, i.e. when $X(x, y) \equiv 0$ and hence $\Phi(\xi, y) \equiv 0$. In that case equation (2.14) reduces to

$$
G_{2}(\xi)=(1-\eta)^{-1}\left\{\zeta^{2} \bar{\Psi}_{p}(\xi,|\xi|)+2 \eta|\xi| \bar{\Psi}(\xi,|\xi|)\right\}
$$

and equation (2.15) to

$$
\sigma_{x x}(x, 0)=\frac{2 \mu}{1-\eta} \mathscr{F}^{-1}\left[\xi^{2} \bar{\Psi}_{p}(\xi,|\xi|)+2 \eta|\xi| \bar{\Psi}(\xi,|\xi|) ; \xi \rightarrow x\right] .
$$


Now if we write

$$
\bar{Y}(\xi, p)=\mathscr{L}[\mathscr{F}\{Y(x, y) ; x \rightarrow \xi\} ; y \rightarrow p],
$$

it is easily shown that

$$
\bar{\Psi}(\xi, p)=\frac{\rho}{2 \mu p} \bar{Y}(\bar{\zeta}, p)
$$

so that equation (3.1) reduces to

$$
\sigma_{x x}(x, 0)=-\frac{\rho}{1-\eta} \mathscr{F}^{-1}\left[(1-2 \eta) \bar{Y}(\xi,|\xi|)-|\xi| \bar{Y}_{p}(\xi,|\xi|) ; \xi \rightarrow x\right] .
$$

Now, by the definition of the Fourier and Laplace transforms,

$$
\bar{Y}(\xi,|\xi|)=\frac{1}{\sqrt{(2 \pi)}} \int_{-\infty}^{\infty} d \alpha \int_{0}^{\infty} Y(\alpha, \beta) e^{-|\xi| \beta+i \xi \alpha} d \beta,
$$

so that

$$
\mathscr{F}^{-1}[\bar{Y}(\xi,|\xi|) ; \xi \rightarrow x]=\frac{1}{\pi} \int_{-\infty}^{\infty} d \alpha \int_{0}^{\infty} \frac{\beta Y(\alpha, \beta)}{\beta^{2}+(x-\alpha)^{2}} d \beta
$$

Similarly we have that

$$
-|\xi| \bar{Y}_{p}(\xi,|\xi|)=\frac{|\xi|}{\sqrt{(2 \pi)}} \int_{-\infty}^{\infty} d \alpha \int_{0}^{\infty} \beta Y(\alpha, \beta) e^{-|\xi| \beta+1 \alpha \xi} d \beta
$$

from which it follows that

$$
-\mathscr{F}^{-1}\left[|\xi| \bar{Y}_{p}(\xi,|\xi|) ; \xi \rightarrow x\right]=\frac{1}{\pi} \int_{-\infty}^{\infty} d \alpha \int_{0}^{\infty} \frac{\beta\left\{\beta^{2}-(x-\alpha)^{2}\right\}}{\left\{\beta^{2}+(x-\alpha)^{2}\right\}^{2}} Y(\alpha, \beta) d \beta
$$

If we substitute these values into equation (3.2) we obtain the relation

$$
\sigma_{x x}(x, 0)=-\frac{2 \rho}{\pi} \int_{-\infty}^{\infty} d \alpha \int_{0}^{\infty} \beta Y(\alpha, \beta) \frac{\beta^{2}+\eta(x-\alpha)^{2} /(\eta-1)}{\left\{\beta^{2}+(x-\alpha)^{2}\right\}^{2}} d \beta
$$

from which to derive the surface stress.

For instance the surface stress due to a body force of total strength $P$ distributed uniformly over the strip $y=h,|x|<a$ and acting normally to the $x$-axis is given by the equation

$$
\sigma_{x x}(x, 0)=\frac{P h}{\pi a} \int_{-a}^{a} \frac{h^{2}+\eta(x-\alpha)^{2} /(\eta-1)}{\left\{h^{2}+(x-\alpha)^{2}\right\}^{2}} d \alpha .
$$

The integration is elementary and we find that

$$
\sigma_{x x}(x, 0)=\frac{P}{2(1-\eta) \pi a}\left\{(1-2 \eta)\left(\theta_{2}-\theta_{1}\right)+\frac{h(x+a)}{r_{2}^{2}}-\frac{h(x-a)}{r_{1}^{2}}\right\},
$$

where $\theta_{1}=\tan ^{-1}\{(x-a) / h\}, \theta_{2}=\tan ^{-1}\{(x+a) / h\}, r_{1}^{2}=(x-a)^{2}+h^{2}$ and $r_{2}^{2}=(x+a)^{2}+h^{2}$. D2 
4. Solution for a point force. Another special case of some interest is that in which a point force $F$ is acting at the point $(0, h),(h>0)$ in the direction of the $x$-axis. We then have

$$
\rho X=F \delta(x) \delta(y-h), \quad \rho Y=0,
$$

so that

$$
\bar{\Phi}(\xi, p)=-\frac{i F}{2 \mu \sqrt{ }(2 \pi)^{-1}} e^{-h p}, \quad \bar{\Psi}(\xi, p)=0 .
$$

From equations (2.15) and (2.16) we find that in this case

$$
\sigma_{x x}(x, 0)=-\frac{F}{\sqrt{(2 \pi)}} \mathscr{F}^{-1}\left[\left(2 i \operatorname{sgn} \xi-\frac{i}{1-\eta} h \xi\right) e^{-h|\xi|} ; \xi \rightarrow x\right],
$$

from which it is easily deduced that

$$
\sigma_{x x}(x, 0)=\frac{2 F x}{\pi\left(h^{2}+x^{2}\right)^{2}}\left[x^{2}-\frac{\eta}{1-\eta} h^{2}\right] .
$$

If we take $F=P \sin \theta$ in this equation and $\rho Y(\alpha, \beta)=-P \cos \theta \delta(\alpha) \delta(\beta-h)$ in equation (3.3), we find that the surface stress due to a point force $P$ acting at the point $(0, h)(h>0)$, in a direction which makes an angle $\theta$ with the negative direction of $y$ is given by the equation

$$
\sigma_{x x}(x, 0)=\frac{2 P}{\pi\left(h^{2}+x^{2}\right)^{2}}\left[h\left(h^{2}-\frac{\eta}{1-\eta} x^{2}\right) \cos \theta+x\left(x^{2}-\frac{\eta}{1-\eta} h^{2}\right) \sin \theta\right] \text {. }
$$

5. Conservative body forces. Finally we consider the special case in which the body forces are conservative, i.e. there exists a function $V(x, y)$ such that

$$
X(x, y)=-\frac{\partial V}{\partial x}, \quad Y(x, y)=-\frac{\partial V}{\partial y}
$$

It is then easily shown that

$$
\bar{\Phi}(\xi, p)=-\Psi(\xi, p)=\frac{\rho}{2 \mu} \bar{V}(\xi, p)
$$

where

$$
\bar{V}(\xi, p)=\mathscr{L}[\mathscr{F}\{V(x, y) ; x \rightarrow \xi\} ; y \rightarrow p] .
$$

If we substitute from equations (5.1) into equation (2.15) and insert the resulting expression in equation (2.16), we find that

$$
\sigma_{x x}(x, 0)=\frac{2(1-2 \eta)}{1-\eta} \rho \mathscr{F}^{-1}[|\xi| \vec{\nabla}(\xi,|\xi|) ; \xi \rightarrow x]
$$

Using the fact that

$$
\bar{V}(\xi,|\xi|)=\frac{1}{\sqrt{ }(2 \pi)} \int_{-\infty}^{\infty} e^{i \xi \alpha} d \alpha \int_{0}^{\infty} V(\alpha, \beta) e^{-|\xi| \beta} d \beta,
$$


and that

$$
\mathscr{F}^{-1}\left\{|\xi| e^{i \xi \alpha-|\xi| \beta} ; \xi \rightarrow x\right\}=\sqrt{\left(\frac{2}{\pi}\right) \frac{\beta^{2}-(x-\alpha)^{2}}{\left[\beta^{2}+(x-\alpha)^{2}\right]^{2}},}
$$

we find that the surface stress is given by the equation

$$
\sigma_{x x}(x, 0)=\frac{2(1-2 \eta)}{\pi(1-\eta)} \rho \int_{-\infty}^{\infty} d \alpha \int_{0}^{\infty} \frac{\left[\beta^{2}-(x-\alpha)^{2}\right]}{\left[\beta^{2}+(x-\alpha)^{2}\right]^{2}} V(\alpha, \beta) d \beta
$$

6. The surface displacement. In a similar way we can derive expressions for the components $u_{x}(x, 0), u_{y}(x, 0)$ of the surface displacement. From equations (2.4) and (2.9) we find that

$$
U_{x}(\xi, y)=i \xi^{-1}\left[\left\{(1-\eta) D^{2}+\eta \xi^{2}\right\} G+(1-\eta) \Phi+\eta \Psi\right]
$$

from which it follows immediately by using equations (2.8) and (2.11) that

i.e. that

$$
U_{x}(\xi, 0)=i \xi^{-1}(1-\eta) G_{2}(\xi),
$$

$$
u_{x}(x, 0)=(1-\eta) \mathscr{F}^{-1}\left\{i \xi^{-1} G_{2}(\xi) ; \xi \rightarrow x\right\} .
$$

If we substitute the expression for $G_{2}(\xi)$ given by (2.15), we find that

$$
\begin{aligned}
u_{x}(x, 0)=\mathscr{F}^{-1}\{2 \eta i \operatorname{sgn} \zeta \bar{\Psi}(\xi,|\xi|) & +i \xi \bar{\Psi}_{p}(\xi,|\xi|) \\
& \left.+2(1-\eta) i \operatorname{sgn} \xi \bar{\Phi}(\xi,|\xi|)+i \xi \bar{\Phi}_{p}(\xi,|\xi|) ; \xi \rightarrow x\right\} .
\end{aligned}
$$

From equations (2.6) and (6.1) we find that

$$
U_{y}(\xi, 0)=\xi^{-2}\left[(1-\eta) G_{3}(\xi)+(1-\eta) \Phi_{y}(\xi, 0)+\eta \bar{\Psi}_{y}(\xi, 0)\right]
$$

and substituting the value of $G_{3}(\xi)$ given by equation (2.14), we find that

$$
u_{y}(x, 0)=\mathscr{F}^{-1}\left[(1-2 \eta) \bar{\Psi}(\xi,|\xi|)-|\xi| \bar{\Psi}_{p}(\xi,|\xi|)+\bar{\Phi}(\xi,|\xi|) ; \xi \rightarrow x\right] .
$$

For instance, if the body forces are derivable from a potential function $V(x, y)$, we find from equations (5.1), (6.2) and (6.3) that

$$
\begin{gathered}
u_{x}(x, 0)=\frac{(1-2 \eta) \rho}{\mu} \mathscr{F}^{-1}[i \operatorname{sgn} \xi \bar{V}(\xi,|\xi|) ; \xi \rightarrow x], \\
u_{y}(x, 0)=\frac{\rho}{2 \mu} \mathscr{F}^{-1}\left[2 \eta \bar{V}(\xi,|\xi|)+|\xi| \bar{V}_{p}(\xi,|\xi|) ; \xi \rightarrow x\right] .
\end{gathered}
$$

The integrations are elementary and we find that

and

$$
u_{x}(x, 0)=\frac{(1-2 \eta) \rho}{\pi \mu} \int_{-\infty}^{\infty}(x-\alpha) d \alpha \int_{0}^{\infty} \frac{V(\alpha, \beta) d \beta}{\beta^{2}+(x-\alpha)^{2}}
$$

$$
u_{y}(x, 0)=\frac{\rho}{2 \mu \pi} \int_{-\infty}^{\infty} d \alpha \int_{0}^{\infty} \beta V(\alpha, \beta) \frac{\left\{(1+2 \eta) \beta^{2}-(1-2 \eta)(x-\alpha)^{2}\right\}}{\left[\beta^{2}+(x-\alpha)^{2}\right]^{2}} d \beta
$$




\section{REFERENCES}

1. E. Melan, Der Spannungszustand der durch eine Einzelkraft im Innern beanspruchten Halbscheibe, Z. Angew. Math. Mech. 12 (1932), 343.

2. I. N. Sneddon, The stress distribution due to a force in the interior of a semi-infinite elastic solid, Proc. Cambridge Philos. Soc. 40 (1944), 229-238.

3. A. E. Green, A note on certain stress distributions in isotropic and aelotropic materials, Proc. Cambridge Philos. Soc. 41 (1945), 224-231.

4. I. N. Sneddon, Fourier transforms (McGraw-Hill, New York, 1951).

UNIVERSITY OF GLASGOW

GLASGOW, W.2 